\section{Band 16, Heft 1, März 2006}

Editorial

5 Gedankensplitter zur Geschichte der

Verhaltenstherapie

Hand, I. (Hamburg)

Originalarbeiten

7 Stressbewältigung am Arbeitsplatz: Ein stationäres Gruppentherapieprogramm zur

Koch, S.; Hedlund, S. (Prien); Rosenthal, S. (Schönau); Hillert, A. (Prien)

17 Dialektisch-behaviorale Therapie der BorderlinePersönlichkeitsstörung in der Regelversorgung - das Saarbrücker Modell

Höschel, K. (Mannheim)

25 Angstsensitivität bei Kindern mit Aufmerksamkeitsdefizit-/Hyperaktivitätsstörung Melfsen, S.; Alpers, G.W.; Walitza, S.; Warnke, A. (Würzburg)

33 Achtsamkeit: Konzeptuelle Aspekte und Ergebnisse zum Freiburger Achtsamkeitsfragebogen

Heidenreich, T. (Frankfurt/M.); Ströhle, G. (Jena); Michalak, J. (Bochum)

43 Erfassung spezifischer Kontrollerwartungen:

Der Fragebogen zu therapiebezogenen

Kontrollerwartungen (TBK)

Delsignore, A.; Schnyder, U. (Zürich); Znoj, H. (Bern)

Übersichtsarbeit

51 Zum Krankheitsbild der generalisierten Angststörung aus heutiger Sicht

Stiglmayr, C; Stiglmayr, Ch. (Berlin); Jerschke, S.; Schehr, K. (Freiburg i.Br.)

Interview

59 Hommage an Johannes C. Brengelmann (1920-1999) drei seiner Schüler über die Aufbruchstimmung der früher 1970er Jahre

Fichter, M.M. (Prien)

65 Neuigkeiten vom Buch- und Zeitschriftenmarkt

69 Fort- und Weiterbildung

72 Mitteilungen der Verbände

77 Tagungen und Kongresse

2 Impressum

U2 Erläuterungen zum Titelbild (2. Umschlagseite) Leibl, C.; Breindl, K.; Naab, S. (Prien/Chiemsee)
Vol. 16, Issue 1, März 2006

Editorial

5 Snatches of Memory on the Development of Behavioral Therapy

Hand, I. (Hamburg)

Original Articles

7 Occupational Stress Management: An Inpatient Group Therapy Program on

Koch, S.; Hedlund, S. (Prien); Rosenthal, S. (Schönau); Hillert, A. (Prien)

17 Dialectical Behavior Therapy for Borderline Personality Disorder in Standard Medical Care - the Saarbrücken

Treatment Program

Höschel, K. (Mannheim)

25 Anxiety Sensitivity in Children with Attention Deficit Hyperactivity Disorder Melfsen, S.; Alpers, G.W.; Walitza, S.; Warnke, A. (Würzburg)

33 Mindfulness: Conceptual Aspects and Results of the Freiburg Mindfulness Inventory Heidenreich, T. (Frankfurt/M.); Ströhle, G. (Jena); Michalak, J. (Bochum)

43 Assessment of Specific Control Expectancies: The Questionnaire on Control Expectancies in Psychotherapy (TBK)

Delsignore, A.; Schnyder, U. (Zürich); Znoj, H. (Bern)

Review Article

51 The Clinical Picture of Generalized Anxiety Disorder from Today's Point of View Stiglmayr, C.; Stiglmayr, Ch. (Berlin); Jerschke, S.; Schehr, K. (Freiburg i.Br.)

Interview

59 Homage to Johannes C. Brengelmann (1920-1999) Three Former Disciples on the Pioneering Spirit of the 1970's

Fichter, M.M. (Prien)

65 Articles and Books

69 Education

72 Information by Behavior Therapy Associations

77 Meetings and Conferences

2 Imprint

C2 Explanations on the Title (Inside front cover) Leibl, C.; Breindl, K.; Naab, S. (Prien/Chiemsee)

\begin{tabular}{ll}
\hline KARGER & @ 2006 S. Karger GmbH, Freiburg \\
Fax +49 761 45207 14 & Artikel (Volltext) und Inhaltsverzeichnisse \\
$\begin{array}{l}\text { E-mail Information@Karger.de } \\
\text { www.karger.com }\end{array}$ & sowie das vorläufige Inhaltsverzeichnis des nächsten Heftes: \\
www.karger.com/ver_bk.htm
\end{tabular}

